\section{Cahiers de Narratologie}

Analyse et théorie narratives

33 | 2018

L'Art du roman chez Umberto Eco

\title{
Le rôle de l'image dans Le Cimetière de Prague
}

\section{Mohamed Bernoussi}

\section{OpenEdition}

\section{Journals}

Electronic version

URL: http://journals.openedition.org/narratologie/8252

DOI: 10.4000/narratologie.8252

ISSN: $1765-307 X$

\section{Publisher}

LIRCES

\section{Electronic reference}

Mohamed Bernoussi, « Le rôle de l'image dans Le Cimetière de Prague », Cahiers de Narratologie [Online], 33 | 2018, Online since 23 July 2018, connection on 01 May 2019. URL : http:// journals.openedition.org/narratologie/8252; DOI : 10.4000/narratologie.8252

This text was automatically generated on 1 May 2019.

Article L.111-1 du Code de la propriété intellectuelle. 


\title{
Le rôle de l'image dans Le Cimetière de Prague
}

\author{
Mohamed Bernoussi
}

1 Le Cimetière de Prague partage avec le roman qui précède cette prédilection pour l'image. Le texte est en effet ponctué pour ainsi dire de gravures et d'illustrations censées jouer le rôle d'illustrations $\mathrm{du}$ texte, mais qui entretiennent des rapports beaucoup plus complexes et semblent soulever de nombreuses questions quant à l'intention de l'auteur, celle de l'œuvre ainsi qu'aux incidences sur sa réception.

2 L'objet de cette étude est d'étudier l'importance de l'image visuelle, plus précisément la gravure, de cerner son rôle et d'interroger ses spécificités par rapport au texte et à l'économie globale de l'œuvre.

3 L'intérêt d'Eco pour l'image est casuel si l'on considère sa production romanesque globalement. Dans Le Nom de la rose, l'image est quasi-absente, mis à part le plan de l'abbaye. Dans ce polar médiéval, le texte jouit de ses pleins droits de parole transcrite sur le papier en défiant le temps et l'espace. Dans le second roman, Le Pendule de Foucauld, nous n'avons pas non plus d'image, mis à part quelques diagrammes et une typographie assez variée puisque trois modèles typographiques cohabitent dans le texte principal qu'est le récit, en plus des exergues et des fichiers de Belbo distincts du texte principal par leur format réduit et servant d'illustration ou d'image textuelle au premier. L'île du jour d'avant n'accorde à l'image aucune importance, le même désintérêt est reconduit dans Baudolino.

4 Il faudra attendre l'avant dernier roman intitulé La dernière flamme de la reine Loana pour voir l'image être réhabilitée auprès du texte, lui servant même de prétexte, car tout le roman tourne autour des tentatives de Yambo pour retrouver la mémoire et les souvenirs du passé à travers des affiches publicitaires, des enseignes et des logos de produits de consommation de toutes sortes.

5 Dans le roman qui nous intéresse, Le Cimetière de Prague, l'image a la même importance, non seulement au niveau quantitatif mais aussi qualitatif. Avant de commencer à décrire et analyser ces images et leur rôle, un bref résumé du roman s'impose, car c'est un roman dense, labyrinthique et tellement difficile à débrouiller qu'Umberto Eco lui-même a pensé 
intégrer à la fin du roman un résumé des événements en forme de colonnes. De quoi s'agit-il?

6 Le Cimetière de Prague raconte l'histoire d'un personnage Simon Simonini, sorte de Baudolino des temps modernes, mais plus cynique et cruel, qui s'illustre dans la falsification des documents en tout genre dans un XIXème siècle féru d'images, de gravures, de magazines, de préjugés racistes et de romans feuilleton. Le portrait de ce falsificateur de génie est le prétexte pour dresser deux fresques historiques croisées de l'Italie et de la France du XIXème siècle, ainsi que de leurs voisins prusses et russes. Simonini joue aussi le rôle de repère dans ce roman labyrinthique. Eco reprend ici nombre d'idées déjà développées dans les romans précédents, comme la contrefaçon, le complot, le parfait faux, etc.

7 Le Cimetière de Prague compte 55 illustrations qui structurent 550 pages de texte, soit à peu près et hormis quelques variantes, une illustration toutes les dix pages. Mises à part trois ou quatre gravures empruntées à des bibliothèques, tout le reste appartient aux archives personnelles du romancier. Toutes les gravures tournent autour d'un seul et unique thème, celui du juif et du complot; elles représentent soit des personnages quelconques soit des personnalités qui ont marqué l'histoire du XIXème siècle français. C'est cette dernière catégorie, nous le verrons plus avant, qui pose problème.

8 Les portraits représentent aussi des personnalités célèbres, qui ont marqué l'histoire française ou italienne, Dreyfus, Garibaldi, etc. à côté de ces figures historiques, nous retrouvons divers types d'illustrations représentant les francs-maçons, Charcot, les carbonari, une loge maçonnique, des Francs-maçons, portrait d'un hystérique, affiche d'époque antimaçonnique, etc. Cette diversité a un dénominateur commun, celui de la falsification et de la conspiration ou du secret. Eco réexploite à nouveau ses lectures de Simmel, déjà utilisées dans Le Pendule de Foucauld ou Baudolino. Précurseur involontaire de Simmel, Simon Simonini sait que le secret constitue une arme redoutable, qu'il peut être utilisé dans les manipulations et les jeux de pouvoir, et que le pire des secrets est le secret vide ou inventé de toutes pièces ; n'est-ce pas lui qui déclare que chercher des preuves est difficile alors que les inventer est plus facile et plus économique. Simonini s'avère cependant être un falsificateur redoutable, car en plus d'être un génie du faux, c'est un antisémite dans la pure tradition du XIXème siècle. De nombreuses gravures font écho à ce que pense Simonini ; elles remplissent donc leur fonction d'éléments d'appui de ce que pense ou aime à penser le personnage, à savoir que les juifs, les francs-maçons et les autres œuvrent à la perte et à la fin de l'humanité. Parfois ces gravures sont reproduites de façon massive et créent quelques déséquilibres entre le texte principal, pour ainsi dire, et les illustrations. Le rapport n'est plus celui d'une simple illustration ou de subordination, mais de concurrence, voire dans certains cas, de superordination. Ce qui ne manque pas de donner à l'œuvre un certain rythme et d'instaurer chez le lecteur quelques réflexes de lecture, y compris une certaine accoutumance au texte visuel. L'importance accordée ici à la gravure s'explique en partie par la technique romanesque d'Umberto Eco, inaugurée depuis Le Nom de la rose, et qui veut que dans tout roman, la sémiosis et l'encyclopédie de son époque soient reproduites. Comme nous l'avons vu dans les chapitres précédents, pour Eco, inscrire un roman dans une époque donnée, c'est reproduire la langue, la sensibilité et le savoir de cette époque de façon précise et fidèle. Or lorsqu'on connaît l'importance de la gravure et de l'illustration dans l'Encyclopédie du XIXème siècle, on comprend pourquoi elle acquiert un statut aussi privilégié dans Le Cimetière de Prague. Mais ce procédé, qui semble être au cœur de l'esthétique romanesque 
d'Eco, a des conséquences singulières sur sa réception et sur son projet initial. Parmi celles-ci, nous en retrouvons quelques-unes relatives à la lecture, car non seulement la multiplication des gravures concurrence le texte en donnant un rythme à l'œuvre et en accoutumant le lecteur à l'image, mais elle présente par moment des stratégies interprétatives qui s'écartent du texte, voire les contredisent. Nous savons depuis Lector in Fabula, en passant par Les Limites de l'interprétation, pour ne citer que les textes les plus importants sur cette question, comment chaque œuvre prévoit un lecteur modèle qu'elle construit à travers des instructions et des stratégies inscrites dans l'œuvre. Or ici les choses se compliquent car, en plus de ces stratégies inscrites dans le texte, les gravures présentent des stratégies inscrites dans les images, plus puissantes et plus directives à en croire Eco lui-même. Dans Kant et l'ornithorynque, le sémioticien écrit ceci à propos des stimuli de perception dans les signes visuels :

«Le stimulus de perception m'empêche de voir (ou d'entendre) du point de vue de ma subjectivité, en tant que corporalité; il ne m'offre qu'un seul profil des choses, et non la multiplicité des profils que la perception actuelle m'offrirait [...] devant les stimuli de substitution, j'ai déjà accepté que quelqu'un ait vu pour moi ». (1991:365)

Dans cette perspective, Le Cimetière de Prague serait le théâtre de deux stratégies interprétatives et de deux instructions différentes, celle d'Umberto Eco comme auteur modèle et celle de l'auteur ou des auteurs des gravures empruntées, ce qui risque de compromettre la stratégie de départ, du moins de la parasiter.

10 Pour illustrer ces distorsions entre stratégies interprétatives textuelles et stratégies interprétatives visuelles, nous avons sélectionné quelques exemples issus du corpus juif. On compte une dizaine de portraits de juifs $(17,81,432,459,513,526)$. Ces portraits se veulent la traduction exacte de ce que pense Simon Simonini des juifs. Ils représentent ces derniers sous des formes repoussantes qui vont dans le sens de la stylistique et de la rhétorique des textes consacrés au sujet et dont l'hyperbole et la redondance constituent les principales figures. Je prendrai comme exemple la gravure de la page 17 et celle de la page 526 vers la fin. 


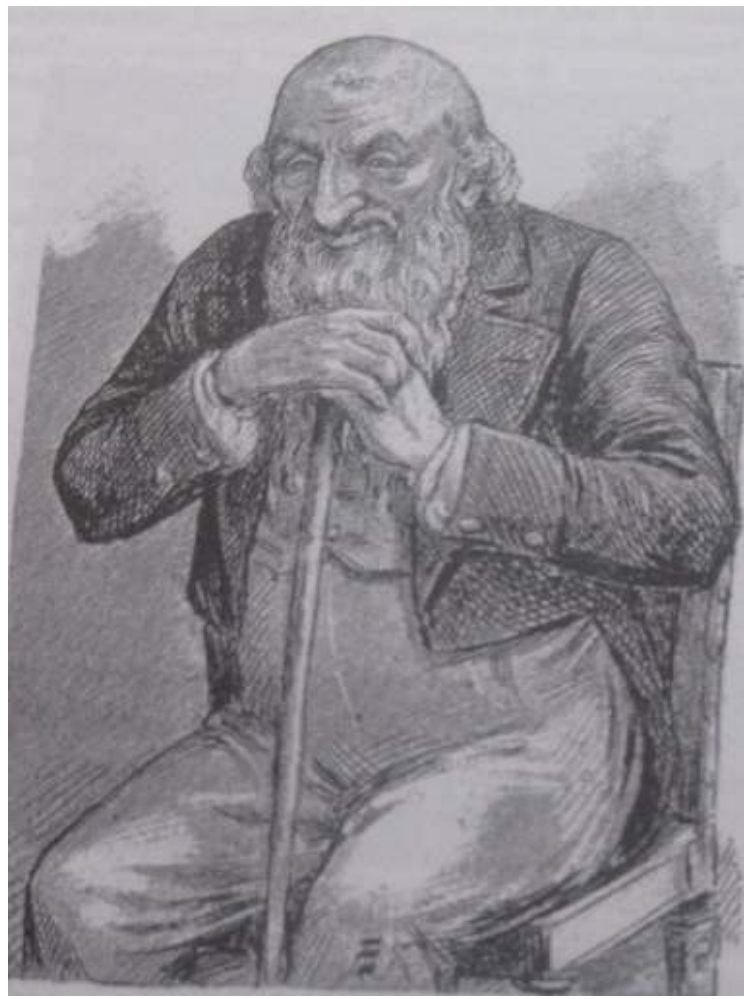

Gravure p.17

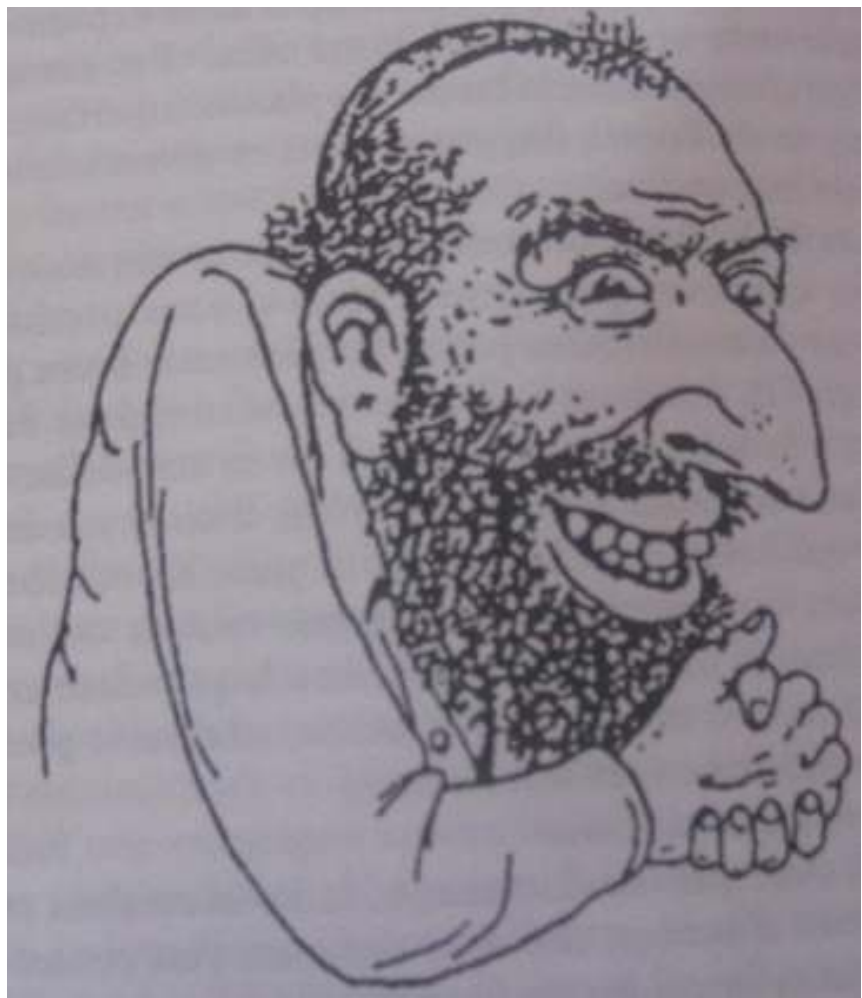

Gravure p.526

Le détail qui attire l'attention dans ces deux portraits du juif est celui du fameux nez et de sa forme démesurée, surtout dans la seconde. Les deux illustrations s'opposent par la densité du trait et du contour dans la seconde et par l'aspect fouillé et subtil dans la 
première. Les deux renvoient en effet à deux imaginaires du juif. La dernière renvoie à un imaginaire panique et anecdotique utilisant des stéréotypes visuels renvoyant au nez démesuré, et aux grosses dents, signes stéréotypés de cupidité et de voracité. La première gravure est beaucoup plus subtile car jouant sur l'ambiguïté des traits et des stimuli de perception, les contours sont un peu flous. Le juif assis, malgré ses airs de grand-père inoffensif, demeure angoissant, même s'il n'y a aucun artifice visuel qui le signifie de façon littérale. Ce type de gravure remplit normalement son rôle d'illustration et vient donc corroborer ce que le texte affirme.

12 Certaines gravures vont cependant jusqu'à parasiter les stratégies de l'auteur modèle ou de l'intentio operis. Ces gravures posent problème comme nous allons le voir, car ce sont des illustrations qui ont un ancrage spatio-temporel spécifique ou historique. Je prendrai deux exemples pour illustrer les problèmes qu'elle pose. Il s'agit de Dreyfus tel qu'il est représenté le jour de sa dégradation par un journal parisien, Le petit journal (459) et le frontispice ou la page de couverture du Protocole des sages de Sion (550).

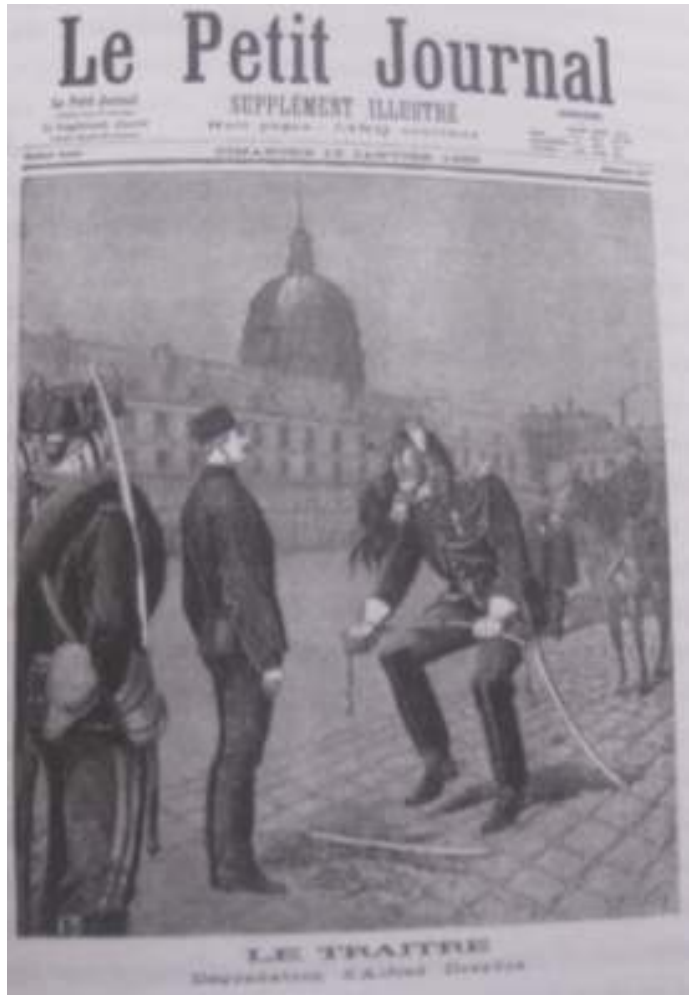

Gravure p. 459 


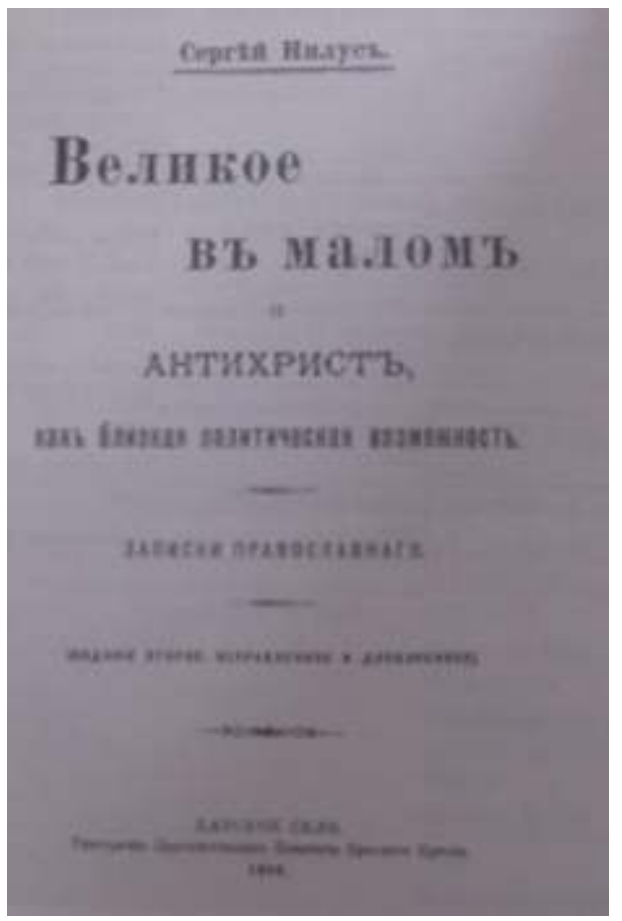

Gravure p. 550

Dans le premier exemple, c'est le rapport entre le contenu du texte et celui de l'image censée le reproduire qui est problématique. Lorsqu'il aborde l'affaire Dreyfus, le texte dit clairement sur plusieurs pages qu'il s'agit d'un complot, d'une machination, etc. Sur plusieurs pages (de 448 à 460) le lecteur apprend comment Drumont, écrivain devenu célèbre pour avoir écrit un livre intitulé La France Juive, a contacté, via Estehrazy, Simonini pour fabriquer un rapport d'espionnage et l'attribuer à un officier appelé Dreyfus pour le piéger ; le but à travers cette machination étant de faire croire comment l'armée française est infiltrée par des juifs qui œuvrent à sa perte. Le texte est donc clair, son isotopie, ses topiques et les divers autres types d'instruction vont dans le sens d'un complot contre l'officier Dreyfus ; mais le choix de l'illustration ne l'est pas, car au lieu d'une illustration qui montrerait par exemple les comploteurs à l'œuvre au moment de la fabrique du faux document, ou de nous renseigner d'une façon ou d'une autre sur la construction de la fabrication de cette trahison, l'image nous montre via un support non négligeable, voire objet de foi, la une du Petit journal. En effet la couverture reproduit une illustration de la destitution de Dreyfus. La force de cette image réside dans le fait qu'elle ne livre que le dénouement qui figure la punition du traitre et pose comme chose admise sa culpabilité, c'est-à-dire la majeure partie du récit, comme une simple évidence à reconstituer par le lecteur. Sa force de persuasion au sens rhétorique réside dans le fait qu'elle manipule les temporalités relatives au récit de l'affaire de Dreyfus en narcotisant plusieurs temporalités clefs dans le récit et en ne présentant que la dernière, celle de la condamnation du traître. Le reste étant sous-entendu comme acquis, évident ou de peu d'importance. C'est un moment difficile pour le romancier, car c'est à ce moment-là qu'il se laisse ou se fait doubler par le point de vue d'un observateur délégué sur l'action en cours. La reproduction de cette page de couverture du Petit journal du 13 Dimanche 1895, qui réfère à un monde possible actuel, celui de la France de 1895, avec le texte d'Umberto Eco où l'on peut lire « ..un géant officier des gendarmes, le casque empanaché, s'approche du capitaine, lui arrache galons, boutons.. », est problématique ; elle donne à voir deux 
mondes possibles qu'il est vain de vouloir considérer en même temps : le monde possible actuel de la gravure qui réfère à celui de 1895 et le monde possible virtuel, celui d'Umberto Eco et de son roman. Il est impossible de les concevoir simultanément. Cela ressemble à un blivet, où l'œil ne peut considérer le dessin en entier sans défier nos capacités mentales et nos sens qui ne peuvent qu'appréhender un élément à la fois.

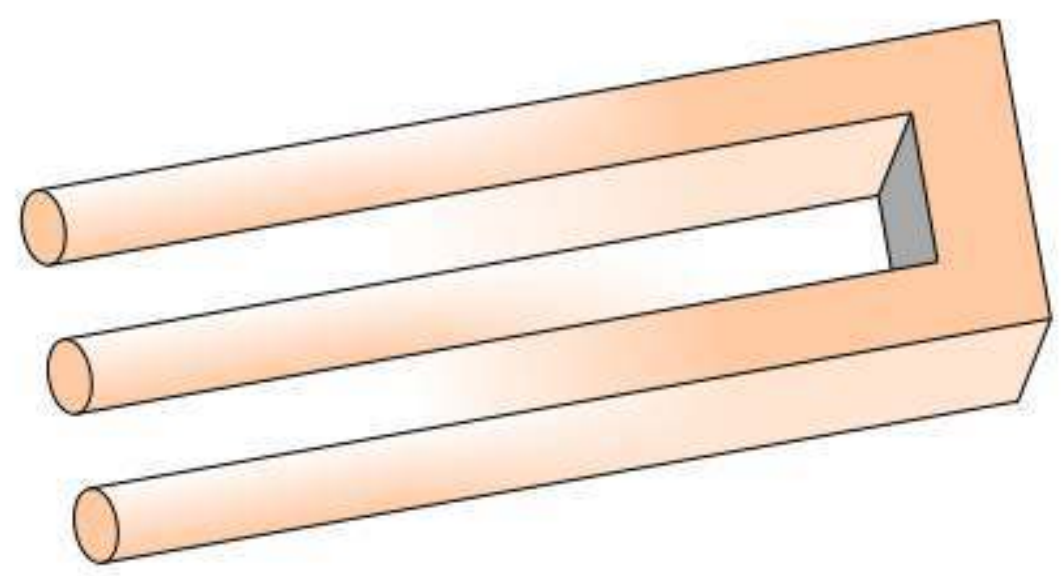

Blivet

Sakurambo CC BY-SA 3.0 https://fr.wikipedia.org/wiki/Blivet - /media/File:Blivet.svg

14 Cette cohabitation de plusieurs mondes possibles, introduite ici par le texte et ses images, soulève une question, déjà bien présente à travers les allusions aux événements historiques dans le texte, propre au statut même du Cimetière de Prague en particulier, et des romans d'Eco en général. Comment un roman qui accorde une place de choix à des références historiques et à des illustrations qui ont valeur de document peut-il prétendre à la littérarité ou à la fiction? Cela n'installe-t-il pas une ambiguïté ?

15 La question a divisé et divise encore la critique. Elle devient cependant plus sensible lorsqu'elle aborde un sujet aussi sensible et aussi surveillé que celui de l'antisémitisme. Le problème réside dans la reproduction de cette littérature antisémite à une échelle aussi importante. On peut repérer deux attitudes, la première estime que devant la masse énorme des références historiques et des références en images comme en texte à l'antisémitisme, le roman d'Eco finit par devenir ambigu, lorsqu'il ne sacrifie pas la littérarité de l'œuvre, car si on enlève toutes ces références et toutes ces illustrations issues du monde actuel, rien ne reste. La seconde attitude consiste à penser qu'il est possible d'utiliser des documents et des personnages antisémites actuels pour nourrir une fable et que cela n'entame en rien la valeur littéraire du roman. Nous pensons de notre côté que si certes ces deux mondes ne peuvent pas se rencontrer ou ne peuvent pas être considérés en même temps, cela ne nous empêche pas de les concevoir dans un espace unique, celui du roman d'Eco. L'image de Dreyfus que nous voyons garde certes son autonomie, mais fait partie désormais d'un monde possible, celui du roman d'Umberto Eco. Cela d'une part, d'autre part, l'intention du texte et par conséquent celle d'Umberto Eco est de raconter le XIXème siècle, sa sémiosis, c'est-à-dire sa langue, sa sensibilité et son encyclopédie. Or, s'il y a un sujet qui domine dans ce siècle, c'est bien celui de l'antisémitisme sous toutes ses formes. 
16 Autre question que soulève la reproduction de ces gravures et illustrations historiques est celle du manque apparent de distance d'Eco vis-à-vis du sujet de l'antisémitisme, et qui a suscité quelques réserves. Il serait réducteur, voire caricatural d'instruire cette interprétation. Ici deux intentions semblent entrer en conflit : l'intentio auctoris, Eco qui a réuni pendant des années ces gravures sur l'antisémitisme, et l'intentio operis, où le texte donne des instructions claires pour rester dans la reproduction et non dans l'apologie de textes antisémites. Sa démarche dans ce roman est beaucoup plus subtile et plus nuancée que cela. Ce qui l'intéresse dans le texte n'est pas le vrai ou le faux, c'est comment le faux en reproduisant les principes du vrai peut devenir vrai. Sa démarche est celle d'un sémioticien qui est persuadé que tout est question de signe et d'interprétation. Eco est persuadé que le vrai ne peut se définir sans le faux, que ce dernier est désigné ainsi, non à cause de ses propriétés internes, mais d'une identité prétendue, que les deux notions sont circulaires, car se définissant mutuellement. Eco recourt à l'histoire et aux illustrations historiques, multipliant ainsi les mondes possibles pour montrer comment les jugements d'authenticité sont le fruit de raisonnements persuasifs, fondés sur des preuves vraisemblables, pas totalement irréfutables et que les hommes peuvent facilement être trompés.

Le discours antisémite arrive à passer pour vrai en reproduisant certains éléments du vrai. Simon Simonini, personnage principal du roman, peut reproduire tous les documents possibles et imaginables; il finit par devenir imbattable dans la fabrication de documents qui sont plus vrais que le vrai. La place de choix des gravures antisémites dans le texte et de documents historiques n'exprime pas le manque de distance d'Eco, elle exprime plutôt sa conviction des pouvoirs de l'image et du signe iconique dans la construction et l'élaboration de la sémiosis antisémite au XIXème siècle.

\section{BIBLIOGRAPHY}

Bernoussi, Mohamed. 1999. «Le Problème du lecteur dans l'œuvre d'Umberto Eco », Semiotica 124-3/4. Berlin/new York, Walter de Gruyter, 255-267.

-2010. «La crise de la représentation entre les phantasmes du sujet et les dérobades de l'objet », Maknasat ${ }^{\circ} 20$, publications de la faculté des Lettres de Meknes.

Eco, Umberto, 1975. Trattato di semiotica generale. Milano: Bompiani.

-((1979) 1985). Lector in fabula. Le rôle du lecteur, Myriem Bouzaher (trad.). Paris : Grasset et Fasquelle.

-((1984) 1988). Sémiotique et philosophie du langage, Myriem Bouzaher (trad.), Paris : P.U.F.

-((1988) 1990). Le Pendule de Foucauld, J.N. Schifano (trad.),Paris, Grasset.

-((2010) 2011). Le Cimetière de Prague, J.N. Schifano (trad), Paris, Grasset. 


\section{ABSTRACTS}

If, in a novel like The Last Flame of Queen Loana, the image is used both in a relevant and happy way to show how Umberto Eco conceives, the subject and its memory, in The Cemetery of Prague, it, memory, is problematic, because it is not always in harmony with the textual strategy of the model author. This is so because the numerous illustrations out of which is composed the novel are meant to fulfill their usual role, but a mindful (an attentive) reading shows that these illustrations entertain with the verbal text complex relationships and raise many questions concerning the author's intention, that of his work and its reception.

Si dans un roman comme La dernière flamme de la reine Loana l'image est utilisée de façon à la fois pertinente et heureuse pour montrer comment Eco conçoit le sujet et sa mémoire, dans Le Cimetière de Prague, elle est problématique, car elle n'est pas toujours en harmonie avec la stratégie textuelle de l'auteur modèle. Les nombreuses illustrations qui composent le roman sont censées remplir leur rôle habituel, mais une lecture attentive montre qu'elles entretiennent avec le texte verbal des rapports complexes et soulèvent de nombreuses questions quant à l'intention de l'auteur, celle de l'œuvre et sa réception.

\section{INDEX}

Mots-clés: cimetière de Prague, antisémitisme, XIXème siècle, pouvoir de l'image, intentio operis

Keywords: Prague Cemetery, Antisemitism, 19th Century, the power of the image, Intentio Operis

\section{AUTHOR}

\section{MOHAMED BERNOUSSI}

Université de Meknès 